\title{
A promising novel formulation for articular cartilage regeneration: Preclinical evaluation of a treatment that produces SOX9 overexpression in human synovial fluid cells
}

\author{
IVAN DELGADO-ENCISO ${ }^{1,2}$, JUAN PAZ-GARCIA ${ }^{3}$, ALEJANDRINA RODRIGUEZ-HERNANDEZ ${ }^{2}$, \\ VIOLETA M. MADRIGAL-PEREZ ${ }^{2}$, ARIANA CABRERA-LICONA ${ }^{4}$, ALEJANDRO GARCIA-RIVERA ${ }^{2}$, \\ ALEJANDRO D. SORIANO-HERNANDEZ ${ }^{1,2}$, JOSE L. CORTES-BAZAN ${ }^{1}$, HECTOR R. GALVAN-SALAZAR ${ }^{1,5}$, \\ JOSE VALTIERRA-ALVAREZ ${ }^{6}$, JOSE GUZMAN-ESQUIVEL ${ }^{5}$, IRAM P. RODRIGUEZ-SANCHEZ ${ }^{7}$, \\ MARGARITA L. MARTINEZ-FIERRO ${ }^{8}$ and BRENDA PAZ-MICHEL ${ }^{4}$ \\ ${ }^{1}$ Cancerology State Institute, Colima State Health Services, Colima 28000; ${ }^{2}$ School of Medicine, \\ University of Colima, Colima 28030; ${ }^{3}$ Centro Hospitalario Union, Villa de Álvarez, Colima 28950; \\ ${ }^{4}$ Esteripharma México, S.A. de C.V., Ciudad de México 03100; ${ }^{5}$ Hospital General de Zona No. 1 IMSS, Villa de Álvarez, \\ Colima 28983; ${ }^{6}$ Hospital Regional Universitario, Colima State Health Services, Colima 28000; ${ }^{7}$ Department of Genetics, \\ School of Medicine, Nuevo Leon Autonomous University, Monterrey, Nuevo León 64460; ${ }^{8}$ Molecular Medicine Laboratory, \\ Academic Unit of Human Medicine and Health Sciences, Universidad Autónoma de Zacatecas, Zacatecas 98160, México
}

Received June 4, 2017; Accepted September 7, 2017

DOI: $10.3892 / \mathrm{mmr} .2017 .8336$

\begin{abstract}
Osteoarthritis (OA) is a chronic disorder of synovial joints, in which there is progressive softening and disintegration of the articular cartilage. OA is the most common form of arthritis, and is the primary cause of disability and impaired quality of life in the elderly. Despite considerable medical necessity, no treatment has yet been proven to act as a disease-modifying agent that may halt or reverse the structural progression of OA. The replacement of the joint with a prosthesis appears to be the best option in the advanced stages of the disease. A formulation (BIOF2) for cartilage regeneration has been recently developed. The present study evaluated the effects of BIOF2 on gene expression in human cell cultures, followed by efficacy trials in three OA animal models. Human synovial fluid cells that were exposed to the formulation exhibited increased transcription factor SOX-9 (SOX9; chondrogenic factor) expression, and decreased mimecan (mineralization inducer) and macrophage-stimulating protein receptor (osteoclastogenic factor) expression. The intra-articular application of BIOF2 in the animal models significantly increased cartilage thickness from 12 to $31 \%$ at 28 days, compared with articular cartilage treated with saline
\end{abstract}

Correspondence to: Dr Brenda Paz-Michel, Esteripharma México, S.A. de C.V., Patricio Sanz 1582, Colonia del Valle Centro, Ciudad de México 03100, México

E-mail:bpaz@esteripharma.com.mx

Key words: osteoarthritis, transcription factor SOX-9, regeneration, treatment, cartilage solution. The articular area and number of chondrocytes additionally increased significantly, maintaining an unaltered chondrocyte $/ \mathrm{mm}^{2}$ proportion. Evaluation of the histological architecture additionally displayed a decrease in the grade of articular damage in the groups treated with BIOF2. In conclusion, BIOF2 has proven to be effective for treating OA in animal models, most likely due to SOX9 overexpression in articular cells.

\section{Introduction}

Osteoarthritis (OA) is a chronic disorder of synovial joints, in which there is progressive softening and disintegration of articular cartilage accompanied by other alterations, including osteophyte growth, cyst formation and subchondral sclerosis (1). It is the most common form of arthritis, and is the primary cause of disability and impaired quality of life in the elderly (2). By the age of $65, \sim 80 \%$ of the population is likely to suffer from this disease (3), and the knee joint is the most frequently affected. No treatment, despite considerable medical necessity, has yet been proven to act as a disease-modifying agent that may halt or reverse the structural progression of OA. Apart from lifestyle modifications, the only available non-surgical treatments are directed at symptoms, primarily to alleviate pain and improve quality of life (4).

In cases of advanced disease, a recommended option is total joint arthroplasty (TJA), replacing the articulation with a prosthesis. However, such surgical treatment is costly (5), and frequently there is a long waiting list. Although the risk of serious adverse events following TJA is low, perioperative risks may be high in elderly patients or in those with comorbidities. Long-term progression may be considered in patients with a prolonged life expectancy (6). 
In recent years, the aforementioned issues have provoked a search for novel drug therapy regimens. In addition to pain management through antidepressants, nonsteroidal anti-inflammatory drugs or opiates, the oral administration of other substances, including glucosamine, chondroitin sulfate, methylsulfonylmethane or collagen hydrolysates, has been studied, demonstrating limited or no benefit. Intra-articular injections of different substances, including hyaluronic acid derivatives or platelet-rich plasma, are being evaluated (7). Stem cell and gene therapy are additional strategies that are currently in development $(8,9)$.

An innovative concept is the creation of novel cartilage in a damaged joint through the administration of bioactive substances that promote chondrogenesis. This idea is based on the fact that the fluid inside the joint contains mesenchymal cells (MSCs) that are able to differentiate into chondrocytes (9). Corticosteroids are one such bioactive substance. When acting alone, corticosteroids may facilitate tissue atrophy and joint destruction; however, when acting in synergy with other factors, they have been demonstrated to increase a number of chondrogenic markers (10), in addition to aiding in the accumulation of extracellular matrix, which is necessary for the formation of cartilage (11). In addition, substances that contain insulin or certain organic acids favor chondrogenesis (12-14).

A formulation for the regeneration of cartilage has recently been developed (15). It is composed of a corticosteroid, insulin and organic acids. The intra-articular application of this bioactive formulation, termed BIOF2 in the present study, is a promising strategy to alleviate and reverse the adverse effects of OA. The present study evaluated the effects of BIOF2 on gene expression in human cell cultures, followed by efficacy trials in in vivo models.

\section{Materials and methods}

$B I O F 2$. The formulation BIOF2 was provided by Esteripharma México S.A de C.V., Ciudad de México, México.

Effect of BIOF2 on gene expression in human synovial fluid cells. Synovial fluid cells were isolated from a sample from the knee of a 58 years old Mexican women with OA who underwent articular lavage as part of her treatment in the Clínica-Hospital Unión in Villa de Álvarez, Colima, México on November, 2015. The patient voluntarily donated the sample and signed a statement of informed consent. A total of $200 \mu 1$ synovial fluid was incubated in Dulbecco's modified Eagle's medium (DMEM; Gibco; Thermo Fisher Scientific, Inc., Waltham, MA, USA) supplemented with $10 \%$ fetal bovine serum (FBS; Gibco; Thermo Fisher Scientific, Inc.) and 1\% penicillin/streptomycin (PS) in a $25 \mathrm{~cm}^{2}(\mathrm{~T}-25)$ cell culture flask, in a $5 \%$ $\mathrm{CO}_{2}$ atmosphere at $37^{\circ} \mathrm{C}$ for $24 \mathrm{~h}$. Washes with sterile $1 \mathrm{X}$ PBS (pH 7.4; Gibco; Thermo Fisher Scientific, Inc.) were performed to remove unattached cells, and DMEM supplemented with $10 \%$ FBS and $1 \%$ PS was added. Cells were cultured until cell outgrowths were well-established. At passage 3, the cells were morphologically homogeneous and exhibited the appearance of fibroblast-like synoviocytes, with the typical bipolar configuration visible under inverse microscopy. Following the same methodology, a previous report stated that $>98 \%$ of the cells obtained expressed surface markers for fibroblasts (16). Low passage (3-6) cell stocks were maintained at $-70^{\circ} \mathrm{C}$. To perform the differential expression experiment, cells were seeded in two T-25 flasks in DMEM supplemented with 10\% FBS. When the cells covered $80 \%$ of the flask surface, the medium was substituted with supplemented DMEM (1\% FBS). BIOF2 was added to one flask $(5 \%)$ and the other flask was used as a reference. The cells were removed $48 \mathrm{~h}$ subsequently for RNA extraction and to verify the alterations in expression caused by $\mathrm{BIOF} 2$ in certain genes of interest.

Total RNA was extracted from the cells using TRIzol ${ }^{\circledR}$ reagent (Thermo Fisher Scientific, Inc.), according to the manufacturer's protocol. Subsequently, $50 \mathrm{ng}$ total RNA was subjected to a one-step reverse transcription-quantitative polymerase chain reaction (RT-qPCR) using the SuperScript ${ }^{\circledR}$ III Platinum ${ }^{\circledR}$ SYBR $^{\circledR}$ Green One-Step RT-qPCR kit (Invitrogen; Thermo Fisher Scientific, Inc.). qPCR was performed using the Roche Light Cycler version 1.5 (Roche Applied Science, Penzberg, Germany). The reaction was performed as follows: $3 \mathrm{~min}$ at $50^{\circ} \mathrm{C}, 5 \mathrm{~min}$ at $95^{\circ} \mathrm{C}$, and 40 cycles $\left(15 \mathrm{sec}\right.$ at $95^{\circ} \mathrm{C}$, $30 \mathrm{sec}$ at $60^{\circ} \mathrm{C}$ and $60 \mathrm{sec}$ at $95^{\circ} \mathrm{C}$ ), followed by $1 \mathrm{~min}$ at $40^{\circ} \mathrm{C}$. Previously described primers were used to amplify GAPDH, macrophage-stimulating protein receptor (MST1R), transcription factor SOX-9 (SOX9), neurogenic locus notch homolog protein 2 (NOTCH2), and mimecan (OGN) (17-21). The human housekeeping gene, GAPDH, was used for internal normalization. To determine the relative expression of the mRNAs, qPCR data were analyzed using the comparative $\Delta \Delta^{\mathrm{Cq}}$ method, as previously described (22). All data were expressed as a relative fold difference compared with the expression in cells unexposed to BIOF2. RT-qPCR analysis was performed in quadruplicate.

OA animal model evaluation. The new formulation, BIOF2, was evaluated for the treatment of OA of the knee in three animal models. A total of two models were created via intra-articular injection of papain in young animals (BALB/c mice and Californian rabbits). The third model consisted of an advanced-age BALB/c mice. This type of mouse has been reported to spontaneously produce OA. Only male animals were used, since it is known that sex hormones serve an important role in the development of OA, with female hormones acting as a protective factor and male hormones exacerbating the disease (23).

Papain-induced OA mouse model. Male BALB/c mice (Envigo, Huntingdon, UK), between 8 and 10 weeks of age and initially weighing $25-28 \mathrm{~g}$, were used in the present study. The mice were divided into two groups of 23 animals/group. The two groups had intra-articular papain administration to the right knee through the patellar ligament, with a $10 \mu 1$ solution of papain, as has previously been described (24). Papain (type IV, double crystallized; $15 \mathrm{U} / \mathrm{mg}$; Sigma-Aldrich; Merck KGaA, Darmstadt, Germany) was used at a concentration of $2.0 \%(\mathrm{w} / \mathrm{v})$ and supplemented with $0.03 \mathrm{M} \mathrm{L}$-cysteine HCI (Sigma-Aldrich; Merck KGaA). A total of 3 weeks subsequently, $10 \mu \mathrm{BIOF} 2$ was administered to the experimental group and $10 \mu \mathrm{l}$ sterile saline solution was administered to the control group. The two groups received intra-articular administration to the right knee. A total of 8 mice from each group were sacrificed on day 7 following BIOF2 injection, followed 
by 7 mice from each group on day 14, and 8 mice from each group on day 28 . The right knees were removed and processed for histopathological analysis.

Papain-induced OA rabbit model. A total of eight 4-month-old Californian rabbits were included in the present study (initially weighing $2.8-3.3 \mathrm{~kg}$ ). The knee joints were subjected to an intra-articular injection of a $500 \mu$ l solution of papain through the patellar ligament, as described above. A total of 21 days following the application of papain, the right knee was subjected to an intra-articular injection of $500 \mu \mathrm{l} \mathrm{BIOF} 2$ through the patellar ligament and the left knee joint was injected with $500 \mu \mathrm{l}$ saline solution. All the rabbits that received the new formulation were sacrificed on day 28 following treatment, and the knees were histologically analyzed.

Spontaneous OA mouse model. A total of 16 18-month-old $\mathrm{BALB} / \mathrm{c}$ mice were included (initially weighing 30-34 g). This strain of mouse has been reported to present spontaneous osteoarthritis starting at 12 months of age. However, the histological alterations have been reported to present variations from one joint to another and from one individual to another (25). Therefore, as it was not known which joint of which mouse may be more damaged, intra-articular injection of the new formulation into the two knees of the 8 mice in the experimental group was decided upon. Saline solution was injected into the two knees of each mouse in the control group, containing 8 mice. Intra-articular application was performed as in the mouse model described above. A total of 28 days following treatment, the mice were euthanized and the knees were histologically analyzed.

The mice were housed in cages, with a maximum of 5 mice/cage. The mice were fed a standard diet (2018S Tekland Global 18\% Protein Rodent Diet; Envigo). The rabbits were housed in individual cages and fed a standard diet (Tekland 8630 Rabbit Diet; Envigo). Light (12-h light/dark cycle), temperature $\left(23^{\circ} \mathrm{C}\right)$ and humidity $(50 \%)$ were controlled for all the animals and they had access to food and water ad libitum. The animals were sacrificed via sodium pentobarbital overdose.

Histopathological analysis. The knee joints were dissected simultaneously and were fixed in solutions of $10 \%$ formaldehyde for 5 days at $24^{\circ} \mathrm{C}$, and subsequently decalcified in $5 \%$ formic acid for 4 days. The specimens were dehydrated in ethanol, embedded in paraffin wax, sectioned (5- $\mu \mathrm{m}$ thick), and stained at $24^{\circ} \mathrm{C}$ for 5,2 and $1 \mathrm{~min}$, respectively with hematoxylin/eosin and toluidine blue. As the degree of toluidine blue staining is influenced by variables, including staining time, all samples were sectioned and stained using stringent protocols to minimize variability. Images of the mouse femoral weight-bearing cartilage were captured. The slices were evaluated via images captured with an Axiocam MRC-5 model digital camera (Zeiss $\mathrm{GmbH}$, Jena, Germany) attached to an AxioPlan $2 \mathrm{M}$ model bright field optical microscope (Zeiss $\mathrm{GmbH}$ ) with a motorized stage and A-plan x5, x10 and $x 40$ objectives (total magnification, x50 for the rabbit cartilage and x100/x400 for the mouse cartilage). Images of the entire sample surfaces were scanned using the Mosai X and Autofocus modules. All images were captured under the same conditions of light and exposure. One blinded pathologist performed the analyses using AxioVision software version 4.0 (Zeiss GmbH, Jena, Germany).

Cartilage thicknesses from the middle part of the femur was determined (5 measurements for each articular cartilage sample). The measurements were made exclusively in the mid-zone, below the surface, in the femoral weight-bearing region. This was performed as previous reports have stated that in papain-induced models, this part is the most severely affected area that exhibits little or no regenerative alterations (26). Cartilage thickness was determined from zones 1 to 3 (the surface layer to the radial zone) (27).

The relative area of the cartilage and the chondrocyte counts were determined using a photomicroscope at a magnification of $\mathrm{x} 400$ from each scanned section (28). The relative area of the cartilage was calculated using AxioVision software, by drawing a circle with a diameter extending from zone 1 to zone 3 . The chondrocytes within the circle were counted. This made it possible to obtain relative articular values, the total number of chondrocytes in that area, and the chondrocytes $/ \mathrm{mm}^{2}$. A total of three circular areas were quantified at the middle part of the femur for each joint.

The histological architecture of the cartilage was evaluated by two blinded observers using a modification of the published mouse scoring system by Chambers et al (29). A score of 0 represented normal cartilage; 1 was a roughened articular surface and small fibrillations; 2 was fibrillation down to the layer immediately below the superficial layer (zone 2) and a degree of loss of surface lamina; 3 was the loss of surface lamina and fibrillations extending down to the calcified cartilage; 4 was major fibrillations and cartilage erosion down to the subchondral bone; 5 was major fibrillations and erosion of $\leq 80 \%$ of the cartilage; and 6 was a loss of cartilage of $>80 \%$.

Statistical analysis. The quantitative data were represented with their mean and standard deviation, while the ordinal data were presented through medians. Mean and median values were used for the descriptive statistics. For the inferential statistics, normal data distribution was first determined using the Kolmogorov-Smirnov test. All the data groups were distributed normally and exhibited homogeneity of variance. Comparisons between two groups was performed using the Student's t-test. The comparison between the groups of mice sacrificed at different times (days 7, 14, and 28) was performed using one-way analysis of variance with post-hoc Tukey honest significant difference test. Due to the small sample size, the Mann-Whitney U test was employed to compare the RT-qPCR values. Mann-Whitney U was additionally used to compare the data of the histological pattern of the cartilage, as it was determined through an ordinal scale [the Chambers et al (29) scoring system]. Statistical tests were performed using IBM SPSS version 20 software (IBM Corp., Armonk, NY, USA). A 95\% confidence interval was used in all the tests, and $\mathrm{P}<0.05$ was considered to indicate a statistically significant difference.

Ethics. The trials complied with the national and international legal and ethical requirements applicable to pre-clinical research. The experimental protocols were approved by 
the research ethics committee of the Instituto Estatal de Cancerología de los Servicios de Salud del Estado de Colima (Colima, México). The animals were manipulated according to institutional guidelines and the Mexican official norm regulating laboratory animal use (no. NOM-062-ZOO-1999), in addition to the Guide for the Care and Use of Laboratory Animals prepared by the National Academy of Sciences of the USA (2011). All animals were sacrificed according to the American Veterinary Medical Association 2013 guidelines for the sacrifice of animals.

\section{Results}

Alterations in expression caused by BIOF2 in human synovial fluid cells. Synovial fluid cells exposed to BIOF2 for $48 \mathrm{~h}$ exhibited increased SOX9 expression by $>2$-fold $(\mathrm{P}=0.02)$ and decreased MST1R and OGN expression by 5- and 3-fold, respectively $(\mathrm{P}=0.02)$. There were no alterations in $\mathrm{NOTCH} 2$ expression compared with unexposed cells (Fig. 1).

In vivo effects on osteoarthritic cartilage thickness. The thickness of cartilage in the medial femur sections of osteoarthritic model animals was studied in the damaged articulation 28 days following the application of BIOF2. In the mouse model of induced articular damage, the intra-articular administration of saline solution caused no significant alterations in cartilage thickness at 28 days. By contrast, BIOF2 application caused increased articular thickness from day 14 of treatment, with a highly significant difference at day 28 , compared with the saline solution group ( $\mathrm{P}<0.01$; Fig. $2 \mathrm{~A})$. In the mouse model of spontaneous articular damage (advanced-age mice) and the OA rabbit model, the cartilage of the joints treated with BIOF2 was significantly thicker compared with the controls $(\mathrm{P}=0.001$ and $\mathrm{P}=0.009$ for the mouse and rabbit models, respectively) (Fig. 2B and C). On day 28 following the intra-articular application of BIOF2, cartilage thickness increased by 29,12 , and $31 \%$, respectively, compared with the controls in the models of induced articular damage in mice, spontaneous damage in mice and induced damage in rabbits.

Structure and composition of articular cartilage in the mouse models. The articular area, total number of chondrocytes and histological architecture of the cartilages were determined (Fig. 3). In the papain-induced articular damage mouse model, BIOF2 application significantly increased the articular area $(\mathrm{P}=0.01)$ and the total number of chondrocytes $(\mathrm{P}=0.04)$ at day 28 , compared with the group treated with saline solution (Fig. 3A and D). The joints treated with saline solution remained unaltered throughout the period following treatment. The area and cellularity increased by an average of 70\% compared with the cartilage treated with saline solution. In the spontaneous OA mouse model, the results were similar, with a $36 \%$ increase in area $(\mathrm{P}=0.04)$ and a $40 \%$ increase in cellularity $(\mathrm{P}=0.009)$, compared with the control group (Fig. 3B and E). It is important to note that the number of chondrocytes $/ \mathrm{mm}^{2}$ remained unaltered in all the groups (Fig. 3C and F).

With respect to the qualitative evaluation of the histological architecture of the cartilage, on day 28 following BIOF2

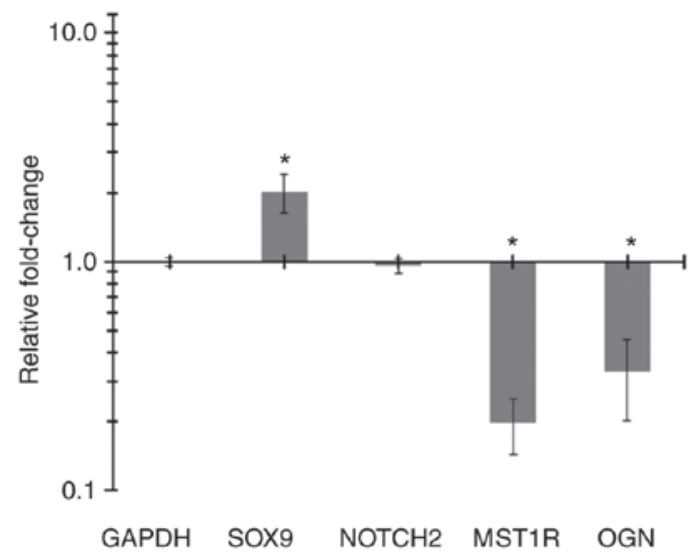

Figure 1. Expression level alterations caused by BIOF2 in exposed and unexposed cells, assayed by reverse transcription-quantitative polymerase chain reaction analysis. In the presence of BIOF2, SOX 9 expression increased and MST1R and OGN levels decreased. NOTCH2 remained unaltered. Data expressed as alterations (log-scale) in mRNA abundance were ratios of mRNAs estimated in BIOF2-exposed synovial fluid cells, compared with unexposed synovial fluid cells under the same conditions. GAPDH was used as an internal control to normalize the target RNA. The graph exhibits the mean and standard deviation of an experiment conducted in quadruplicate at 48 h following exposure. ${ }^{*} \mathrm{P}<0.05$ vs. unexposed cells. SOX 9 , transcription factor SOX-9; NOTCH2, neurogenic locus notch homolog protein 2; MST1R, macrophage stimulating protein receptor; OGN, mimecan.

application there was a significantly decreased grade of articular damage, compared with cartilage treated with saline solution (Fig. 4). According to the scoring system developed by Chambers et al (29), which has a scale from grades 0 to 6 ( 0 , no damage; $6,>80 \%$ loss of cartilage), the medians of the groups treated with $\mathrm{BIOF} 2$ compared with saline solution were 2 vs. $3.5(\mathrm{P}=0.02)$ and 3 vs. $4(\mathrm{P}=0.04)$ for the spontaneous $\mathrm{OA}$ and induced OA mouse models, respectively.

\section{Discussion}

The present study demonstrated that hyaline cartilage regeneration may be induced in vivo via the intra-articular application of the novel bioactive formulation BIOF2. This was observed in three different animal models. In a 28-day follow-up of one of the animal models it was observed that the thickness of the cartilage and the number of chondrocytes began to increase slightly on day 14 post-treatment. However, the most important histological alterations occurred between days 14 and 28, and the latter day was when all the morphometric parameters were highly significant and beneficial in regard to treatment with BIOF2, compared with saline solution application. It was notable that the increase in thickness and articular area was accompanied by a proportional increase in cellularity (chondrocytes $/ \mathrm{mm}^{2}$ was unaltered). This demonstrated that cartilage growth was not solely due to extracellular matrix growth.

In addition, upon analyzing the histological architecture, the joints treated with BIOF2 exhibited a lower grade of damage compared with the controls, suggesting repair towards a normal joint morphology. An additional relevant aspect in relation to the BALB/c mouse animal models, compared with their controls, was the fact that there was a greater increase in cartilage thickness (29\%) in the induced 

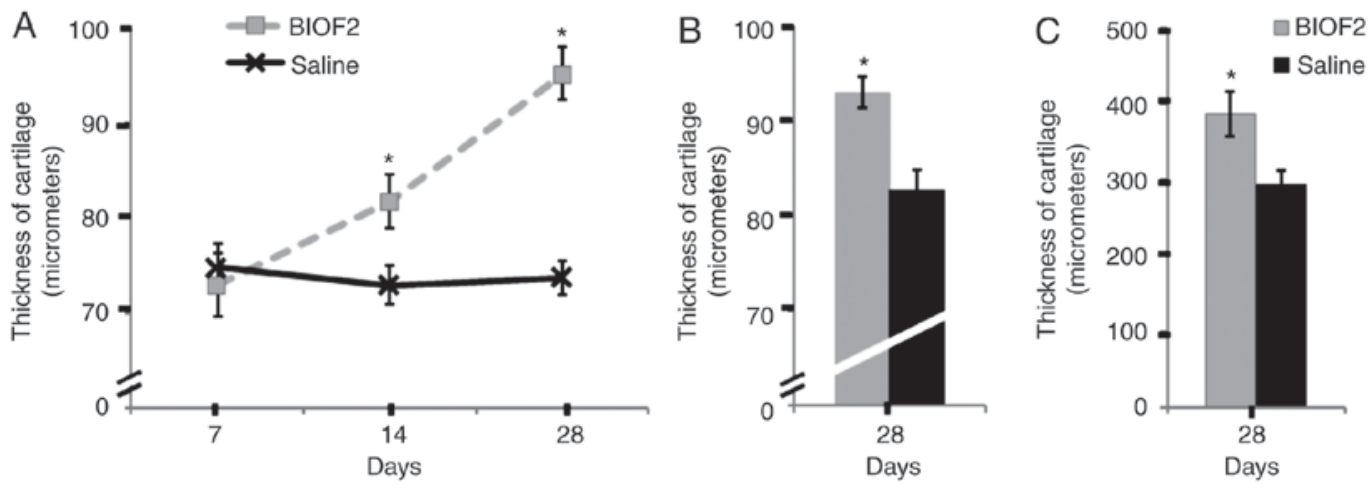

Figure 2. Evaluation of femoral articular cartilage thickness in three different animal models. (A) Mouse model of papain-induced articular damage. Saline solution did not cause alterations in the cartilage during the analysis period; BIOF2 produced a significant increase in thickness at 14 and 28 days following application. (B) Advanced-age mouse model of spontaneous articular damage. (C) Rabbit model of papain-induced articular damage. Treatment with BIOF2 produced a significant increase in articular thickness in the advanced-age mice and the rabbits, compared with respective control groups. The graph exhibits the mean \pm standard deviation. ${ }^{*} \mathrm{P}<0.01$ vs. saline.
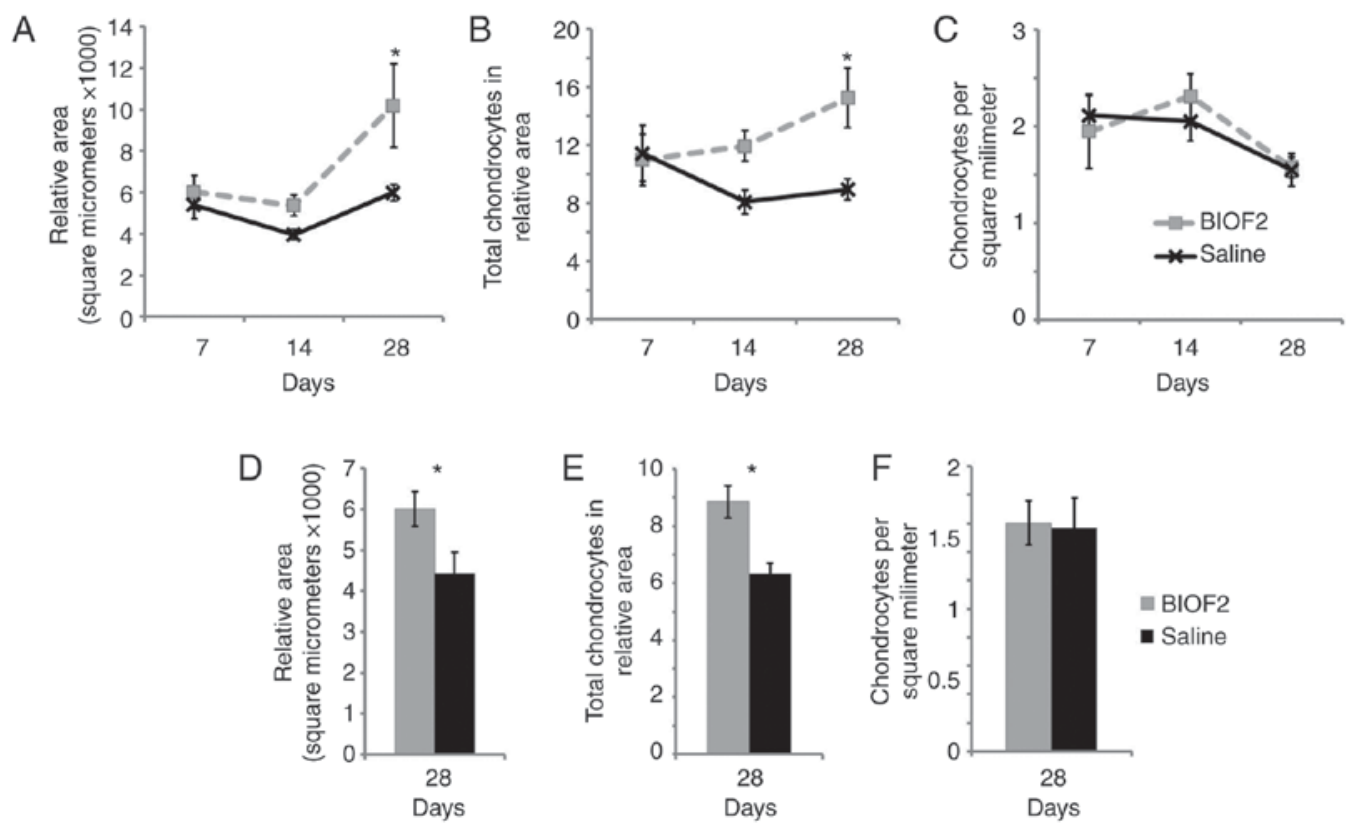

Figure 3. Relative area of the femoral articular cartilage and number of chondrocytes. In the mouse model of papain-induced articular damage, only the post-treatment day 28 evaluation of the BIOF2 treated group exhibited a statistically significant increase in (A) relative area of cartilage and (B) number of chondrocytes. (C) There was no significant difference in the number of chondrocytes $/ \mathrm{mm}^{2}$. Saline solution did not produce any significant alterations in the cartilage during the time of evaluation. In the advanced-age mouse model of spontaneous articular damage evaluated on post-treatment day 28 , BIOF2 produced a significant increase in (D) the area and (E) the number of chondrocytes compared with the control group. (F) There was no significant difference in the number of chondrocytes $/ \mathrm{mm}^{2}$. The graphs exhibit the mean \pm standard deviation. "P<0.05 vs. saline.

articular damage model in young mice compared with the advanced-age mouse model (12\%) at post-treatment day 28 . This concurs with clinical findings that have demonstrated that current techniques for damaged cartilage repair appear to produce better results in young patients (30). This may be relevant to the suggested dosage for young or elderly patients in future clinical trials.

The mechanism through which BIOF2 may regenerate articular cartilage was examined at the molecular level via experiments performed on human synovial fluid cells. BIOF2 caused an increase in the expression of $\mathrm{SOX} 9$, a transcription factor that is essential for chondrocyte differentiation and cartilage formation (31). SOX9 serves a notable role in the development and maintenance of the chondrogenic phenotype.
One previous study demonstrated that SOX9 expression was relatively high in normal cartilage, although its transcript levels were substantially decreased in OA, accompanied by degradation of the extracellular matrix; the study additionally suggested that a reduction of SOX9 transcript levels in osteoarthritic chondrocytes may be responsible for such loss of the extracellular matrix (32). Effective chondrogenesis and inhibition of bone morphogenetic protein 2-induced osteogenesis and endochondral ossification have additionally been demonstrated to be achieved by directing MSCs towards the chondrocyte lineage with SOX9 (33). A further hypothesis for correcting OA is the overexpression of SOX9 through gene therapy, possibly combined with cell therapy $(34,35)$. This indicates that the increase in SOX9 by BIOF2 is a consistent 


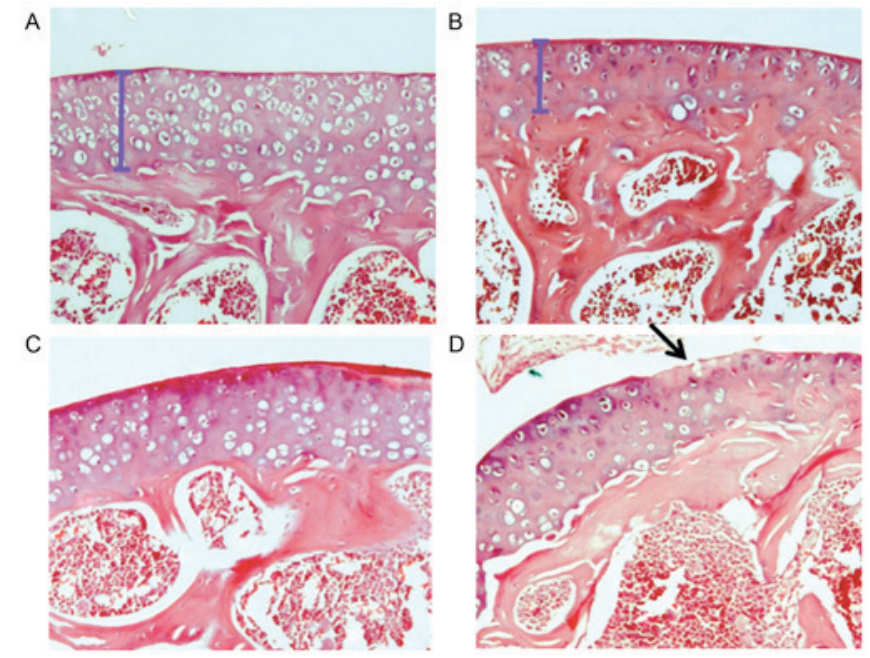

Figure 4. Microscopic images from the medial femur sections of mode mice on treatment day 28. (A) Papain-induced osteoarthritis treated with BIOF2; small and isolated fibrillations are observed over the articular matrix (grade 1). (B) Papain-induced osteoarthritis treated with saline solution; fibrillation is observed immediately below the superficial layer, in addition to the loss of the lamina surface within calcification spots of articular cartilage (grade 2). (C) Advanced-age spontaneous osteoarthritis treated with BIOF2; small and isolated fibrillations are observed in the articular matrix (grade 1) (D) Advanced-age spontaneous osteoarthritis treated with saline solution; a deflated and irregular articular surface (indicated by an arrow), in addition to extended fibrillation and calcification spots, are observed (grade 3). Cartilage thickness of medial femur sections is exhibited in A and B. Hematoxylin and eosin staining; magnification, $\mathrm{x} 200$. Lesions graded according to the Chambers et al (29) scoring system.

mechanism with the therapeutic effect observed in the present study.

The results of the present study demonstrated that BIOF2 caused a decrease in OGN and MST1R expression. OGN has been reported to be elevated in OA synovial fluid samples and may induce the mineralization and calcification of cartilage (37). Additionally, MST1R has been previously identified to be associated with osteoclastogenesis, osteolysis and inflammation $(38,39)$. The decreased expression of these genes is consistent with a therapeutic effect of BIOF2 on OA. Although previous studies have demonstrated that the Notch pathway is active during chondrogenesis $(40,41)$, the present study identified no increase in NOTCH2. However, NOTCH2 expression was measured only once, and the possibility of its modification at another evaluation time may not be excluded, which is a limitation of the present analysis. Gene expression alterations (including SOX9 and cytokines) in the joint tissue following in vivo application of BIOF2 are an important aspect to study in future experiments in animal models or in clinical trials.

The results of the present study have led to the proposal of an innovative strategy to clinically repair osteoarthritic lesions through BIOF2-induced cartilage regeneration. This therapeutic strategy is novel and different from other OA treatment alternatives, including viscosupplementation, gene therapy or cell therapy. The novel strategy is based on modifying the intra-articular microenvironment to stimulate articular regeneration, by generating molecular and morphological alterations in synovial fluid cells and chondrocytes. The synovium is considered to be a candidate source of cells for cartilage. Compared with MSCs from other sources, synovium-derived stem cells have a higher capacity for chondrogenic differentiation (42). Fibrous synovium cells (with the typical bipolar configuration), including the cells cultivated in the present study, have previously been observed to release large numbers of MSCs (43). It is likely that BIOF2 produces molecular alterations, including SOX9 upregulation, in synovial MSCs that favor SOX9 migration to the cartilage and differentiation into chondrocytes. In addition, BIOF2 appears to generate SOX9 elevation in chondrocytes, thus reversing the alterations associated with OA.

There are numerous problems associated with current OA treatment strategies. In advanced disease cases, the recommendation is to substitute the joint with a prosthesis. However, the surgical treatment is costly (5), with long waiting lists, and it may be risky for elderly patients or those with comorbidities (6). Conservative treatment with oral medications, including glucosamine, chondroitin sulfate, methylsulfonylmethane or collagen hydrolysates, has exhibited limited or no benefit. Intra-articular injections of different substances are additionally being evaluated. The intra-articular administration of corticosteroids produces a short-term improvement. However, the repeated use of corticosteroids may facilitate tissue atrophy, joint destruction or cartilage degeneration (44). Hyaluronic acid derivatives produce apparent effectiveness 5-13 weeks following treatment. They are inferior to steroids in the short-term, although they provide greater improvement over an increased length of time (45). Platelet-rich plasma is under consideration as an innovative and promising tool with an effectiveness pattern comparable to the intra-articular administration of hyaluronic acid (7). Stem cell therapy is an additional strategy whose aim is to support the process of self-healing of the knee joint cartilage damage resulting from OA symptoms (1). The intra-articular application of stem cells, facilitating their differentiation into chondrocytes, is a process that includes the separation of the cells by centrifugation and other purification steps, with the aim of increasing cartilage buildup. Clinical data on the effectiveness of stem cell therapy remains insufficient and certain authors have expressed concerns regarding the issues of dosage, intervention timing, mode, route of delivery and the type of stem cells in clinical studies $(8,9)$. It is hypothesized that the BIOF2-induced regeneration strategy has the potential to ameliorate the problems or limitations of current OA treatments.

In conclusion, the application of BIOF2 was effective for the treatment of OA in animal models, possibly as a result of increased SOX9 expression in articular cells. This regeneration strategy merits further study as a realistic research focus in the near future, particularly in clinical trials.

\section{Acknowledgements}

The present study was completed using equipment resources obtained through grant nos. 270485 and 272792 from the INFRAESTRUCTURA-CONACYT-2016 and FOSISS-CONACYT-2016, respectively. Dr Juan Paz and Dr Brenda Paz-Michel declare that they are the inventors of the experimental formulation used in the present study (patent no. US9089580 B1). 


\section{References}

1. Vaishya R, Pariyo GB, Agarwal AK and Vijay V: Non-operative management of osteoarthritis of the knee joint. J Clin Orthop Trauma 7: 170-176, 2016.

2. Karsdal MA, Michaelis M, Ladel C, Siebuhr AS, Bihlet AR, Andersen JR, Guehring H, Christiansen C, Bay-Jensen AC and Kraus VB: Disease-modifying treatments for osteoarthritis (DMOADs) of the knee and hip: Lessons learned from failures and opportunities for the future. Osteoarthritis Cartilage 24: 2013-2021, 2016.

3. Raeissadat SA, Rayegai SM, Hassanbadi H, Fathi M, Ghorbani E, Babaee M and Azma K: Knee osteoarthritis injection choices: Platelet-rich plasma (PRP) verses hyaluronic acid (A one year randomized clinical trial). Clin Med Insights Arthritis Musculoskelet Disord 8: 1-8, 2015.

4. Bannuru RR, Osani M, Vaysbrot EE and McAlindon TE: Comparative safety profile of hyaluronic acid products for knee osteoarthritis: A systematic reviewand network meta-analysis. Osteoarthritis Cartilage 24: 2022-2024, 2016.

5. Herrera-Espiñeira C, Escobar A, Navarro-Espigares JL, Castillo Jde D, García-Pérez L and Godoy-Montijano A: Total knee and hip prosthesis: Variables associated with costs. Cir Cir 81: 207-213, 2013.

6. Hawker GA, Badley EM, Borkhoff CM, Croxford R, Davis AM, Dunn S, Gignac MA, Jaglal SB, Kreder HJ and Sale JE: Which patients are most likely to benefit from total joint arthroplasty? Arthritis Rheum 65: 1243-1252, 2013.

7. Montañez-Heredia E, Irízar S, Huertas PJ, Otero E, Del Valle M, Prat I, Díaz-Gallardo MS, Perán M, Marchal JA and Hernandez-Lamas Mdel C: Intra-Articular injections of Platelet-Rich plasma versus hyaluronic acid in the treatment of osteoarthritic knee pain: A randomized clinical trial in the context of the Spanish national health care system. Int J Mol Sci 17: pii:E1064, 2016

8. Richter W: Cell-based cartilage repair: Illusion or solution for osteoarthritis. Curr Opin Rheumatol 19: 451-456, 2007.

9. Uth K and Trifonov D: Stem cell application for osteoarthritis in the knee joint: A minireview. World J Stem Cells 6: 629-636, 2014.

10. Jakobsen RB, Østrup E, Zhang X, Mikkelsen TS and Brinchmann JE: Analysis of the effects of five factors relevant to in vitro chondrogenesis of human mesenchymal stem cells using factorial design and high throughput mRNA-profiling. PLoS One 9: e96615, 2014.

11. Tangtrongsup S and Kisiday JD: Effects of dexamethasone concentration and timing of exposure on chondrogenesis of equine bone Marrow-derived mesenchymal stem cells. Cartilage 7: 92-103, 2016.

12. Scioli MG, Bielli A, Gentile P, Cervelli V and Orlandi A: Combined treatment with platelet-rich plasma and insulin favours chondrogenic and osteogenic differentiation of human adipose-derived stem cells in three-dimensional collagen scaffolds. J Tissue Eng Regen Med 11: 2398-2410, 2017.

13. Yao Y, Zhai Z and Wang Y: Evaluation of insulin medium or chondrogenic medium on proliferation and chondrogenesis of ATDC5 cells. Biomed Res Int 2014: 569241, 2014.

14. Gerstenfeld LC, Toma CD, Schaffer JL and Landis WJ Chondrogenic potential of skeletal cell populations: Selective growth of chondrocytes and their morphogenesis and development in vitro. Microsc Res Tech 43: 156-173, 1998.

15. Paz-García J and Paz-Michel BA: Formulation for regeneration of bone, cartilage, teeth, and periodontium and treatment of tumors and cysts. US Patent US9089580 B1. Filed July 28, 2015; issued February 4, 2015.

16. Casnici C, Lattuada D, Tonna N, Crotta K, Storini C, Bianco F, Truzzi MC, Corradini C and Marelli O: Optimized 'in vitro' culture conditions for human rheumatoid arthritis synovial fibroblasts. Mediators Inflamm 2014: 702057, 2014.

17. Li XW, Tuergan M and Abulizi G: Expression of MAPK1 in cervical cancer and effect of MAPK1 gene silencing on epithelial-mesenchymal transition, invasion and metastasis. Asian Pac J Trop Med 8: 937-943, 2015.

18. Mayer S, Hirschfeld M, Jaeger M, Pies S, Iborra S, Erbes T and Stickeler E: RON alternative splicing regulation in primary ovarian cancer. Oncol Rep 34: 423-430, 2015.

19. Biason-Lauber A, Konrad D, Meyer M, DeBeaufort C and Schoenle EJ: Ovaries and female phenotype in a girl with $46, X Y$ karyotype and mutations in the CBX2 gene. Am J Hum Genet 84: 658-663, 2009.
20. O'Neill CF, Urs S, Cinelli C, Lincoln A, Nadeau RJ, León R, Toher J, Mouta-Bellum C, Friesel RE and Liaw L: Notch2 signaling induces apoptosis and inhibits human MDA-MB-231 xenograft growth. Am J Pathol 171: 1023-1036, 2007.

21. Shin JE, Oh JH, Kim YK, Jung JY and Chung JH: Transcriptional regulation of proteoglycans and glycosaminoglycan chain-synthesizing glycosyltransferases by UV irradiation in cultured human dermal fibroblasts. J Korean Med Sci 26: 417-424, 2011.

22. Pottosin I, Delgado-Enciso I, Bonales-Alatorre E, Nieto-Pescador MG, Moreno-Galindo EG and Dobrovinskaya O: Mechanosensitive $\mathrm{Ca}^{2+}$-permeable channels in human leukemic cells: Pharmacological and molecular evidence for TRPV2. Biochim Biophys Acta 1848: 51-59, 2015.

23. Ma HL, Blanchet TJ, Peluso D, Hopkins B, Morris EA and Glasson SS: Osteoarthritis severity is sex dependent in a surgical mouse model. Osteoarthritis Cartilage 15: 695-700, 2007.

24. van der Kraan PM, Vitters EL, van de Putte LB and van den Berg WB: Development of osteoarthritic lesions in mice by 'metabolic' and 'mechanical' alterations in the knee joints. Am J Pathol 135: 1001-1014, 1989.

25. Stoop R, van der Kraan PM, Buma P, Hollander AP, Billinghurst RC, Poole AR and van den Berg WB: Type II collagen degradation in spontaneous osteoarthritis in C57B1/6 and BALB/c mice. Arthritis Rheum 42: 2381-2389, 1999.

26. Moriizumi T, Yamashita N and Okada Y: Papain-induced changes in the guinea pig knee joint with special reference to cartilage healing. Virchows Arch B Cell Pathol Incl Mol Pathol 51: 461-474, 1986.

27. Hardin JA, Cobelli N and Santambrogio L: Consequences of metabolic and oxidative modifications of cartilage tissue. Nat Rev Rheumatol 11: 521-529, 2015.

28. Fazelipour S, Tootian Z, Matini E and Hadipour-Jahromy M: Histomorphometric alteration of knee articular cartilage and serum alkaline phosphatase in young female mice by chronic supplementation with soybean. Phytother Res 25: 886-891, 2011.

29. Chambers MG, Cox L, Chong L, Suri N, Cover P, Bayliss MT and Mason RM: Matrix metalloproteinases and aggrecanases cleave aggrecan in different zones of normal cartilage but colocalize in the development of osteoarthritic lesions in STR/ort mice. Arthritis Rheum 44: 1455-1465, 2001.

30. Musumeci G, Loreto C, Castorina S, Imbesi R, Leonardi R and Castrogiovanni P: Current concepts in the treatment of cartilage damage. A review. Ital J Anat Embryol 118: 189-203, 2013.

31. Bi W, Deng JM, Zhang Z, Behringer RR and de Crombrugghe B: Sox9 is required for cartilage formation. Nat Genet 22: 85-89, 1999.

32. Goldberg VM, Buckwalter J, Halpin M, Jiranek W, Mihalko W, Pinzur M, Rohan B, Vail T, Walker P, Windsor R and Wright T: Recommendations of the OARSI FDA Osteoarthritis Devices Working Group. Osteoarthritis Cartilage 19: 509-514, 2011.

33. Liao J, Hu N, Zhou N, Lin L, Zhao C, Yi S, Fan T, Bao W, Liang X, Chen H, et al: Sox9 potentiates BMP2-induced chondrogenic differentiation and inhibits BMP2-induced osteogenic differentiation. PLoS One 9: e89025, 2014.

34. Tao K, Frisch J, Rey-Rico A, Venkatesan JK, Schmitt G, Madry H, Lin J and Cucchiarini M: Co-overexpression of TGF- $\beta$ and SOX9 via rAAV gene transfer modulates the metabolic and chondrogenic activities of human bone marrow-derived mesenchymal stem cells. Stem Cell Res Ther 7: 20, 2016.

35. Wan R, Hu J, Zhou Q, Wang J, Liu P and Wei Y: Application of co-expressed genes to articular cartilage: New hope for the treatment of osteoarthritis (Review). Mol Med Rep 6: 16-18, 2012.

36. Balakrishnan L, Nirujogi RS, Ahmad S, Bhattacharjee M, Manda SS, Renuse S, Kelkar DS, Subbannayya Y, Raju R, Goel R, et al: Proteomic analysis of human osteoarthritis synovial fluid. Clin Proteomics 11: 6, 2014.

37. De Ceuninck F, Marcheteau E, Berger S, Caliez A, Dumont V, Raes M, Anract P, Leclerc G, Boutin JA and Ferry G: Assessment of some tools for the characterization of the human osteoarthritic cartilage proteome. J Biomol Tech 16: 256-265, 2005.

38. Akiyama M, Nakahama K and Morita I: Impact of docosahexaenoic acid on gene expression during osteoclastogenesis in vitro-a comprehensive analysis. Nutrients 5: 3151-3162, 2013.

39. Cully M: Bone diseases: MST1R inhibitor prevents bone osteolysis. Nat Rev Drug Discov 16: 164-165, 2017. 
40. Watanabe M, Tezuka Y, Matsuno K, Miyatani S, Morimura N, Yasuda M, Fujimaki R, Kuroda K, Hiraki Y, Hozumi N and Tezuka K: Suppresion of differentiation and proliferation of each chondrogenic cells by Notch. J Bone Miner Metab 21: 344-352, 2003.

41. Hardingham TE, Oldershaw RA and Tew SR: Cartilage, SOX9 and Notch signal in chondrogenesis. J Anat 209: 469-480, 2006.

42. Pei M, He F and Vunjak-Novakovic G: Synovium-derived stem cell-based chondrogenesis. Differentiation 76: 1044-1056, 2008.

43. Katagiri K, Matsukura Y, Muneta T, Ozeki N, Mizuno M, Katano $\mathrm{H}$ and Sekiya I: Fibrous synovium releases higher numbers of mesenchymal stem cells than adipose synovium in a suspended synovium culture model. Arthroscopy 33: 800-810, 2017.
44. Richards MM, Maxwell JS, Weng L, Angelos MG and Golzarian J: Intra-articular treatment of knee osteoarthritis: From anti-inflammatories to products of regenerative medicine. Phys Sportsmed 44: 101-108, 2016.

45. Rodriguez-Maerchan EC: Intra articular injection of hyaluronic acid and other drugs in knee joint. HSS J 9: 180-182, 2013.

This work is licensed under a Creative Commons Attribution-NonCommercial-NoDerivatives 4.0 International (CC BY-NC-ND 4.0) License. 